\title{
Discordance between the QuantiFERON-TB Gold In-Tube and Tuberculin Skin Test: need for a further step?
}

\author{
To the Editor:
}

Screening for latent tuberculosis infection (LTBI) in patients with immune-mediated inflammatory diseases (IMID), candidates for treatment with biological agents, is currently recommended by several national and international guidelines. In addition to the older tuberculin skin test (TST), in the past decade, in vitro interferon- $\gamma$ release assays (IGRAs), such as the QuantiFERON-TB Gold In-Tube (QFT) (Qiagen, Hilden, Germany) and T-SPOT.TB (TSPOT) (Oxford Immunotec, Abingdon, UK), have also been available for diagnosing LTBI. IGRAs are supposed to be less affected by prior vaccination with bacille Calmette-Guérin (BCG) or exposure to non-tuberculous mycobacteria than the TST, resulting in a higher specificity of IGRAs compared to the TST $[1,2]$. Due to the lack of a gold standard, the diagnostic accuracy of IGRAs and TSTs cannot be definitely established. Data from the literature show a reduced sensitivity in immunocompromised patients and a low predictive value for progression to active TB for both TST and IGRA [1-4]. In the last years, several studies evaluated the accuracy of the IGRA and TST in IMID populations, but the findings are conflicting [5]. In this context, different LTBI screening strategies have been recommended $[5,6]$. Considering the suboptimal sensitivity of the available tests, and according to data from a previous study showing a good association of both the QFT and TST with LTBI risk factors, our centre used a screening strategy based on the simultaneous performance of the QFT and TST, in addition to a chest radiograph (CXr) [7]. This strategy, also supported by the European Centre for Disease Prevention and Control guidance, is meant to maximise the sensitivity in immune-compromised individuals, and is especially suited for areas where BCG coverage is low [8]. However, in a considerable number of cases, these tests provide discordant results, giving way to uncertain interpretation in clinical practice. To address this issue, we assessed the performance of a second level of LTBI screening tests for IMID patients, who are candidates for biological agents, if TST/QFT discordant results should occur.

From June 2011 to January 2013, we analysed 369 patients. Among them, 45 resulted with discordant results at baseline (QFT, TST, CXr). After 14-21 days of the baseline tests, the patients progressed to the second step of screening, according to their pattern of discordance (TST+/QFT-; TST-/QFT+; or TST-/QFT-/CXr+): all the enrolled patients underwent further QFT and TSPOTs, whereas a two-step TST was performed only in patients with a negative TST (TST-/QFT+; or TST-/QFT-/CXr+). Demographic information, data on underlying diseases, treatment regimens in the last 3 months and BCG vaccination status were collected by a standardised interview.

The TST was performed by intradermal injection of a 2-TU dose of purified protein derivative (PPD) (PPDRT23; Staten Serum Institute, Copenhagen, Denmark) on the volar side of the forearm by trained physicians; the result was read after 48-72 $\mathrm{h}$ using the ballpoint method, and interpreted in accordance with the published guidelines, with a cut-off of 5 or $10 \mathrm{~mm}$, based on the individual risk of TB infection and progression [9].

Blood samples for the QFT and TSPOT were obtained immediately before performing the TST. Both tests were performed, following the manufacturer's assay kit recommendations, by the same laboratory staff who were unaware of the patients' characteristics and previous results $[10,11]$.

Chest radiographic findings consistent with previous TB include calcified nodular densities or fibrous scarring in the mid/upper lung zones of the lung parenchyma, and pleural thickening.

Informed consent from each patient and local Ethical Committee approval were obtained.

Data from the interviews and tests results were entered into Microsoft Excel 2010 software (Microsoft, Redmond, WA, USA). The agreement between the TSPOT and baseline tests was evaluated using Cohen's $k$, with $k>0.75$ representing excellent agreement, $0.40-0.75$ representing good agreement, and $<0.40$ representing poor agreement [12].

The results of the baseline and second-level screening tests and the demographic and clinical features of the 45 enrolled patients are shown in table 1 . At baseline screening, 11 patients scored TST+/QFT-, seven scored TST-/QFT+ and 27 scored TST-/QFT - with radiological findings suggestive of previous TB at the chest radiograph. Overall, five (11\%) out of 45 were BCG-vaccinated, three of whom were in the TST+/QFTgroup and two were in the TST-/QFT-/CXr+ group. At the second level screening, in the TST+/QFTgroup, six (55\%) out of 11 were TSPOT-positive, whereas all the repeated QFT were confirmed negative. 
In the TST-/QFT+ group, four (57\%) out of seven reverted the previous QFT result and all of them had a QFT-value $<1 \mathrm{IU} \cdot \mathrm{mL}^{-1}$ at baseline (range $0.38-0.65 \mathrm{IU} \cdot \mathrm{mL}^{-1}$ ); one out of seven converted the TST result with the two-step test and none scored positive with the TSPOT. In the TST-/QFT-/CXr+ group, three out

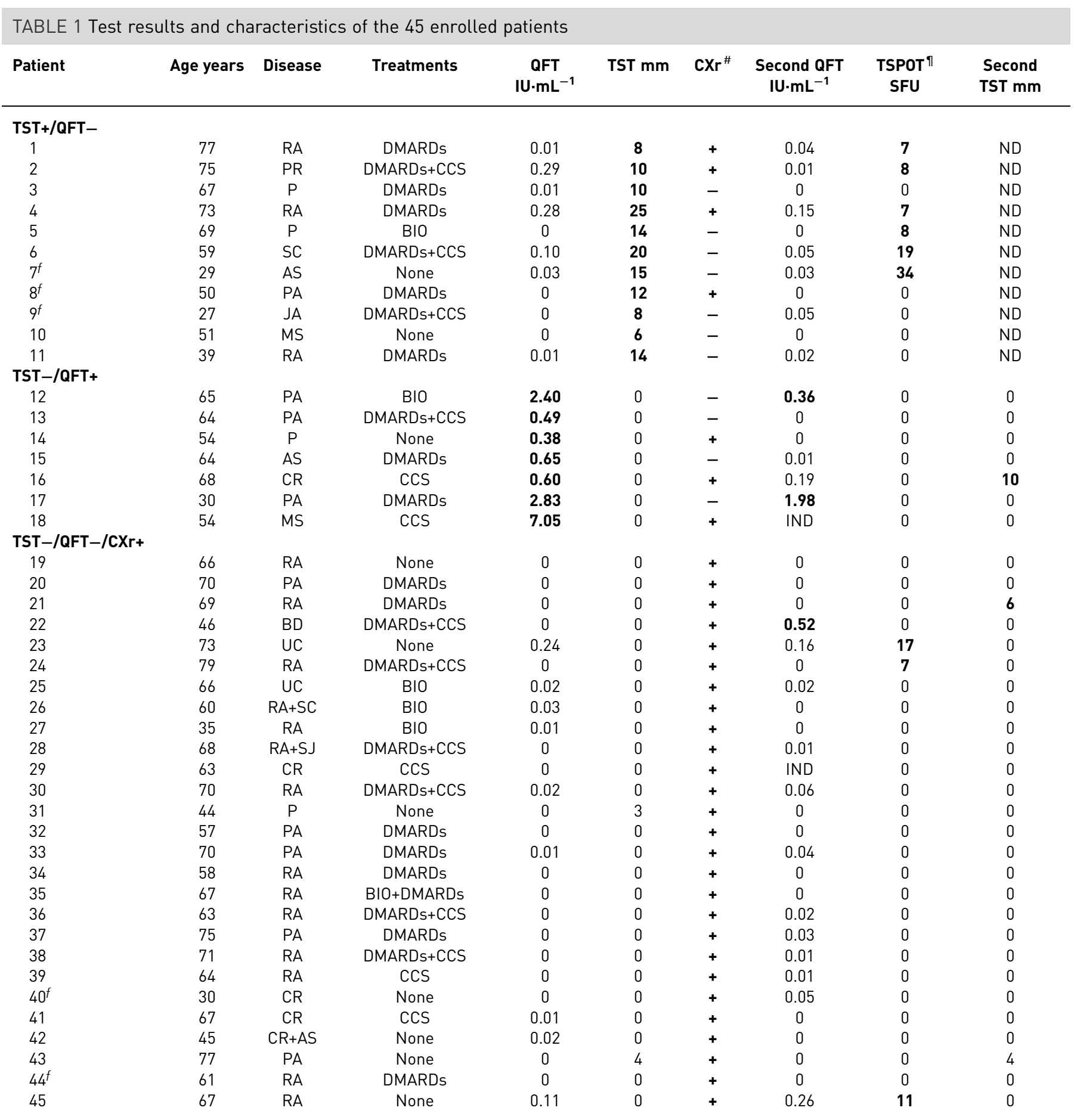

QFT: QuantiFERON-TB Gold In-Tube (Qiagen, Hilden, Germany); IU: international units; TST: tuberculin skin test; CXr: chest radiograph; TSPOT: T-SPOT.TB (Oxford Immunotec, Abingdon, UK); RA: rheumatoid arthritis; PR: polymyalgia rheumatica; P: psoriasis; SC: scleroderma; AS: ankylosing spondylitis; PA: psoriatic arthritis; JA: juvenile idiopathic arthritis; MS: multiple sclerosis; CR: Crohn's disease; BD: Behçet's disease; UC: ulcerative colitis; SJ: Sjögren's syndrome; DMARDs: disease modifying antirheumatic drugs; CCS: corticosteroids; BIO: biological agents; IND: indeterminate; ND: not done. ${ }^{\#}$ : with (+) or without (-) findings consistent with previous tuberculosis; ${ }^{\text {I }}$ : data are reported as the highest SFU value of ESAT- 6 or CFP-10 antigen minus the SFU value of the negative control; ${ }^{f}$ : vaccinated with bacille Calmette-Guérin. Positive results are highlighted in bold. 
of 27 patients scored positive with the TSPOT, one out of 27 with the QFT and one out of 27 with the two-step TST.

Overall, the agreement between baseline tests and TSPOT results was good for the TST $(k 0.49)$ and poor for the QFT $(k-0.21)$.

Interestingly, the TSPOT showed a greater agreement with the TST than the QFT in all the groups. More than half (55\%) of the TST+/QFT- patients scored positive to the TSPOT, suggesting that these results may be associated with LTBI rather than a false-positive result, especially in patients without previous BCG vaccination. Among the three TST+/QFT - patients with a history of BCG vaccination, one scored positive with the TSPOT. The interpretation of this pattern of discordance in BCG-vaccinated patients is critical: as known, the impact of BCG on the TST is strongly affected by the age when it was administered and the time passed since the vaccination [13]. The patients' clinical history and risk factors for TB should be taken into consideration, and more sensitive tests for LTBI detection, such as the TSPOT, may be helpful in this setting. In contrast, repeated QFTs did not lead to conversions. Data from QFT+/TSTpatients confirm the reproducibility issues reported for QFT results around the cut-off value. All the four QFT+/TST - patients with a QFT index value $<0.65 \mathrm{IU} \cdot \mathrm{mL}^{-1}$, reverted their QFT results. A recent systematic review on QFT reproducibility showed a substantial variability for repeated QFTs, even when tests are conducted under the same conditions; since the discrepancy was greater for individuals with initial values in the borderline range $\left(0.25-0.80 \mathrm{IU} \cdot \mathrm{mL}^{-1}\right)$, the authors advise interpreting such QFT results with caution [14]. To note, the median of QFT index values of concordantly positive patients from our population (32 out of 369 ) was $4.70 \mathrm{IU} \cdot \mathrm{mL}^{-1}(95 \%$ CI $2.83-6.56)$.

In the TST-/QFT-/CXr+ group, five (19\%) out of 27 showed positivity with one of the second-level tests, whereas the remaining $81 \%$ confirmed the negative results of the baseline tests. The high rate of negative concordant results of the former and the latter tests may be related to the low specificity of the chest radiograph in differentiating signs of previous TB in which $M$. tuberculosis is already cleared, and lesions where $M$. tuberculosis is still present, though quiescent. Indeed, previous studies have shown that up to $85 \%$ of calcified lesions in the upper lobes of the lungs are sterile, and that the T-cell immunity response declines over time [15]. However, patients with TST-/QFT-/CXr+ are generally considered at lower risk of $\mathrm{TB}$ reactivation and, unless a severe immune-suppression is present at baseline, they are addressed to clinical and radiological follow-up, without recommendation for LTBI treatment before starting the biological agents. In this study, $19 \%$ of the positive results were unmasked through the second-level testing, suggesting that further investigations may detect patients with indication for LTBI treatment, even when there is TST-/QFT-/CXr+ discordance.

The main limitation of the study is the small sample of patients; moreover, control groups of positive and negative concordant patients have not been included.

In conclusion, the study supports the complexity of LTBI diagnosis in the context of the IMID population, due to the patients' characteristics and the imperfect accuracy of the available tests. The simultaneous use of both the QFT and TST, as well as resorting to further exams in the case of discordant results, is advised in their routine LTBI screening. A further step of testing in patients with discordant results may be helpful when confounding factors, such as BCG vaccination or QFT values around the cut-off coexist. In these terms, the use of the TSPOT seems to be more useful than the two-step TST to investigate discordant results and point out otherwise undetected immunological responses. Finally, it's advisable that in the near future, new highly predictive biomarkers will be identified and new assays will be developed to definitely increase the accuracy of LTBI screening in this population.

@ERSpublications

T-SPOT.TB is useful in discordant QFT/TST immune-mediated inflammatory diseases patients candidates for biological drugs http://ow.ly/OZsLx

Michele Spinicci ${ }^{1}$, Jessica Mencarini ${ }^{1}$, Delia Goletti ${ }^{2}$, Antonia Mantella ${ }^{1}$, Nunzia Della Malva ${ }^{3}$, Alessandro Bartoloni ${ }^{1,4}$ and Filippo Bartalesi ${ }^{4}$

${ }^{1}$ Clinica Malattie Infettive, Università degli Studi di Firenze, Florence, Italy. ${ }^{2}$ Unità di Ricerca Traslazionale, Epidemiologia Clinica, Istituto Nazionale di Malattie Infettive (INMI) "Lazzaro Spallanzani”, Rome, Italy. ${ }^{3}$ SOD Microbiologia, Azienda Ospedaliero-Universitaria Careggi, Florence, Italy. ${ }^{4}$ SOD Malattie Infettive e Tropicali, Azienda Ospedaliero-Universitaria Careggi, Florence, Italy.

Correspondence: Filippo Bartalesi, SOD Malattie Infettive e Tropicali, Azienda Ospedaliero-Universitaria Careggi, Largo Brambilla 3, 50134 Florence, Italy. E-mail: bartalesif@aou-careggi.toscana.it

Received: March 122015 | Accepted after revision: June 102015 | First published online: July 232015

Conflict of interest: None declared. 
Acknowledgements: We would like to thank Andrea Baker, English mother tongue speaker, whose work has been very helpful in editing this paper.

\section{References}

1 Pai M, Denkinger CM, Kik SV, et al. Gamma interferon release assays for detection of Mycobacterium tuberculosis infection. Clin Microbiol Rev 2014; 27: 3-20.

2 Diel R, Goletti D, Ferrara G, et al. Interferon- $\gamma$ release assays for the diagnosis of latent Mycobacterium tuberculosis infection: a systematic review and meta-analysis. Eur Respir J 2011; 37: 88-99.

3 Sester M, van Leth F, Bruchfeld J, et al. Risk assessment of tuberculosis in immunocompromised patients. A TBNET study. Am J Respir Crit Care Med 2014; 190: 1168-1176.

4 Sañé Schepisi M, Parracino MP, Mammone A, et al. Immune status and serial Quantiferon-TB Gold In-tube screening for latent Mycobacterium tuberculosis infection among HIV-infected persons in a country with a low tuberculosis incidence. J Infect Dis 2015; 211: 1852-1853.

5 Smith R, Cattamanchi A, Steingart KR, et al. Interferon- $\gamma$ release assays for diagnosis of latent tuberculosis infection: evidence in immune-mediated inflammatory disorders. Curr Opin Rheumatol 2011; 23: 377-384.

6 Denkinger CM, Dheda K, Pai M. Guidelines on interferon- $\gamma$ release assays for tuberculosis infection: concordance, discordance or confusion? Clin Microbiol Infect 2011; 17: 806-814.

7 Bartalesi F, Vicidomini S, Goletti D, et al. QuantiFERON-TB Gold and the TST are both useful for latent tuberculosis infection screening in autoimmune diseases. Eur Respir J 2009; 33: 586-593.

8 European Centre for Disease Prevention and Control. Use of interferon-gamma release assays in support of TB diagnosis. Stockholm, ECDC, 2011.

9 Gardam MA, Keystone EC, Menzies R, et al. Anti-tumour necrosis factor agents and tuberculosis risk: mechanisms of action and clinical management. Lancet Infect Dis 2003; 3: 148-155.

10 Cellestis. QuantiFERON -TB Gold. http://www.quantiferon.com/IRM/pub/ErrorPageNotFound.aspx?aspxerrorpath=/ $\mathrm{irm} /$ content/quantiferon-tb-gold1.aspx Date last accessed: December 1, 2014.

11 OxfordImmunotec. T.SPOT1.TB. www.oxfordimmunotec.com/T-SPOT.TB_International Date last accessed: December 1, 2014.

12 Fliess JL. The measurement of inter-rate agreement. In: Bradley RA, Levin B, Cho Paik M, eds. Statistical methods for rates and proportions. New York, John Wiley \& Sons Inc, 1981; pp. 212-236.

13 Farhat M, Greenaway C, Pai M, et al. False-positive tuberculin skin tests: what is the absolute effect of BCG and non-tuberculous mycobacteria? Int J Tuberc Lung Dis 2006; 10: 1192-1204.

14 Tagmouti S, Slater M, Benedetti A, et al. Reproducibility of interferon gamma release assays: a systematic review. Ann Am Thorac Soc 2014; 11: 1267-1276.

15 Mack U, Migliori GB, Sester M, et al. LTBI: latent tuberculosis infection or lasting immune responses to M. tuberculosis? A TBNET consensus statement. Eur Respir J 2009; 33: 956-973.

\section{Phenotyping chronic pulmonary aspergillosis by cluster analysis}

To the Editor:

Chronic pulmonary aspergillosis (CPA) is a complex disorder involving various underlying conditions and risk factors, clinical and radiological features, and natural histories or responses to treatment [1]. Untreated, patients with CPA have $\geqslant 50 \% 5$-year mortality $[2,3]$. Recently, it was proposed that CPA includes simple aspergilloma, chronic cavitary pulmonary aspergillosis (CCPA) and chronic necrotising pulmonary aspergillosis (CNPA) [1].

However, aside from simple aspergilloma, the presentation and treatment of which is distinct from that of CCPA and CNPA, considerable overlap seems to exist between the various clinical, radiological and histological presentations of CCPA and CNPA $[1,4]$. Improving the classification of CCPA and CNPA by integrating the multiple aspects of the disease is critical for future clinical trials. Here, we used a subject-centred multivariate clustering approach without a priori assumptions to identify phenotypes among patients with CPA (excluding simple aspergilloma) based on integrated clinical, biological and radiological features.

This study was based on a retrospective analysis of 127 patients with CPA (simple aspergilloma excluded) seen between January 2002 and December 2011 in eight chest departments at French university hospitals. The study was approved by the local research Ethics Committee (DR-2012-304) of Poitiers, France. 